\title{
Evaluation of the Solid/Liquid Interface Undercooling During Sn-Se
}

\section{Eutectic Growth}

\author{
M. Zanotello, C.T. Rios, R.H.B. Jacon, R. Caram \\ Universidade Estadual de Campinas, \\ C.P. 6122, 13083-970 Campinas - SP, Brazil \\ Phone: +55-19-788-7966, Fax: +55-19-289-3722 \\ e-mail: caram@fem.unicamp.br
}

Received: January 6, 1998; Revised: May 21, 1998

\begin{abstract}
The Sn-Se eutectic solidification allows one to obtain a lamellar structure, formed by SnSe and $\mathrm{SnSe}_{2}$ compounds, which are $\mathrm{p}$ and $\mathrm{n}$ semiconducting types, respectively. The $\mathrm{SnSe}-\mathrm{SnSe} 2$ eutectic composite is a promising material to be used in photovoltaic device manufacturing. In a lamellar eutectic microstructure, the main parameter, which governs many of its characteristics, is the lamellar spacing. Such a parameter is primarily a result of the undercooling at the solid/liquid interface, which depends on the growth rate and the eutectic system properties. In this work, the Sn-Se alloy corresponding to eutectic composition was studied by using DSC thermal analysis and directional solidification at several growth rates in a vertical Bridgman-Stockbarger unit. The objective of the experiments was to investigate the influence of the growth rate on the growth undercooling, as well as on the eutectic microstructure. The microstructure analysis showed that a very regular and aligned structure formed by the $\mathrm{SnSe}$ and $\mathrm{SnSe}_{2}$ solid phases can be produced. By using the classic eutectic growth theory developed by Jackson and Hunt, the experimental results obtained led to the evaluation of a relationship among growth rates, eutectic growth temperature and lamellar spacing.
\end{abstract}

Keywords: semiconductors, crystal growth, microstructure

\section{Introduction}

In recent decades, eutectic alloy growth has been the subject of several theoretical and experimental studies ${ }^{1-4}$. This research was performed as a result of the possibility of achieving composite materials directly from the liquid phase during eutectic solidification. If a eutectic alloy is directionally grown, a regular structure consisting of two solid phases can be formed: the $\alpha$ and $\beta$ phases. This phenomenon is a result of a cooperative growth of an oriented and an anisotropic structure, where both solid phases grow side by side. This type of solid growth depends on the simultaneous mass transfer in the liquid near the solid/liquid interface. While the $\alpha$ phase segregates the component $\mathrm{B}$, the $\beta$ phase rejects the component $\mathrm{A}$. This process results in a solute increase in the liquid ahead of the $\alpha$ and $\beta$ phases and hence, to lateral mass transport due to the composition gradients of A and B. Lateral mass transport is fundamental to the eutectic growth process.
In eutectic microstructure, the main parameter is the distance between solid phases, as it controls many of its properties. In the case of a lamellar eutectic growth, this parameter is termed "lamellar spacing" and it fundamentally depends on the solid/liquid interface undercooling. The melt undercooling depends on the solute distribution in the liquid near the interface which is connected to the growth rate, to the diffusion process and to some eutectic system properties. The solute distribution in the liquid near solid/liquid interface is associated with the mass transport due to the displacement of the interface and to the mass transport due to atomic diffusion given by Fick's law. A variation in the melt solute distribution leads to a variation in the liquid undercooling, which modifies the interphase spacings ${ }^{1}$.

By solving the diffusion problem at the solid/liquid interface during eutectic growth, Jackson and Hunt ${ }^{1}$ were able to develop a theory of regular eutectic growth. Assuming that the $\alpha$ and $\beta$ solid phase interfaces are flat, they formulated a relationship among the interface undercool- 
ing, the solute distribution field and the interface curvature. The undercooling associated with the solute composition departure from eutectic composition is determined from the phase diagram. The undercooling associated with the curvature at the solid/liquid interface is related to the capillarity effect, and it is calculated by using the Gibbs- Thomson relationship ${ }^{4}$. The total solid/liquid interface undercooling $(\Delta \mathrm{T})$ as a function of both the growth rate $(\mathrm{V})$ and the lamellar spacing $(\lambda)$ is given by the relationship ${ }^{1}$ :

$$
\Delta \mathrm{T}=\mathrm{K}_{1} \lambda \mathrm{V}+\frac{\mathrm{K}_{2}}{\lambda}
$$

where $K_{1}$ and $K_{2}$ are constants determined from the phase diagram and thermodynamic data.

Analysis of Eq. 1 reveals that the total undercooling is not unique, as it depends on the product between the interphase spacing and the growth rate. This fact leads to the assumption that another relationship is essential in order to evaluate the eutectic growth conditions ${ }^{1}$. The solution of this problem was found by applying the hypothesis used by Zener ${ }^{5}$ and Tiller ${ }^{2}$, which can be described by the extremum criterion. According to the extremum criterion, eutectic growth occurs with the minimum liquid undercooling at solid/liquid interface or with the maximum growth rate. So, if the first derivative of the total undercooling is set equal to zero, the following relationships among $\Delta \mathrm{T}, \lambda$ and $\mathrm{V}$, are obtained $^{1,4}$ :

$$
\begin{aligned}
& \lambda^{2} \mathrm{~V}=\frac{\mathrm{K}_{2}}{\mathrm{~K}_{1}} \\
& \frac{\Delta T}{\sqrt{V}}=2 \sqrt{K_{1} K_{2}}=K_{3}
\end{aligned}
$$

$$
\Delta \mathrm{T} \lambda=2 \mathrm{~K}_{2}
$$

In the $\mathrm{Sn}$-Se system, a eutectic transformation allows one to produce the $\mathrm{SnSe}$ and $\mathrm{SnSe}_{2}$ phases which exhibit interesting electronic and optical properties, and hence, this eutectic microstructure is potentially useful in the production of solar cells ${ }^{6-11}$. While tin monoselenide shows an orthorhombic structure and is a p-type semiconductor ${ }^{9}$, the tin diselenide presents a hexagonal $\mathrm{CdI}_{2}$-type crystal structure and is n-type semiconductor ${ }^{9}$. Bhatt et al. have investigated photovoltaic properties of thin films $\mathrm{SnSe}-\mathrm{SnSe}_{2}$ junctions ${ }^{11}$. Based on the properties of the $\mathrm{SnSe}$ and $\mathrm{SnSe}_{2}$ compounds, they suggested that these materials may be applied in the production of photovoltaic devices. Taking into account that a special characteristic of the Sn-Se system is a eutectic reaction that may simultaneously give rise to $\mathrm{SnSe}$ and $\mathrm{SnSe}_{2}$ phases, a promising way of manufacturing low-priced electronic devices using a single growth process is the use of directional growth of the Sn-Se eutectic alloy ${ }^{12}$.
A regular eutectic microstructure is obtained if its phases are arranged side by side and aligned with the direction of the heat flux, the amount of eutectic imperfections is low, and the spacings, for a given growth rate, is distributed over a narrow range of values.

Concerning the directional growth of the Sn-Se eutectic alloy $^{13}$, in spite of the fact that the average interphase spacing with the growth rate variation follows the Jackson and Hunt theory ${ }^{1}$, given by Eq. 2, an intriguing phenomenon was observed. Keeping the growth rate constant, it was observed that the lamellar spacings are scattered over a large range of values ${ }^{13}$. It was also noted that the growth rate variation produces changes in the spacing distribution $^{13}$. It was found that an increase in the growth rate leads to a narrower distribution of spacings.

Bearing in mind that to obtain any electronic device from $\mathrm{Sn}$-Se eutectic microstructure, it is necessary to obtain a very regular microstructure, which makes essential to understand its eutectic reaction in detail. The main aim of this paper is to analyze the influence of the growth conditions on the interfacial undercooling and the microstructure during Sn-Se eutectic growth.

\section{Experimental Procedure}

The effect of the growth conditions on the solid/liquid interface undercooling was investigated by using two different techniques: differential scanning calorimetry analysis $^{14}$ and directional solidification method ${ }^{15}$.

The Sn-Se eutectic alloy was prepared by using a correct quantity of $99.99 \%$ pure Se pellets and $99.999 \%$ pure Sn shot, related to $50.96 \mathrm{wt} \%$ Se. Quartz tubes $8.0 \mathrm{~mm}$ ID x $10 \mathrm{~mm}$ OD and $550.0 \mathrm{~mm}$ in length were sealed at one end and used as the material for encapsulating the alloy. The ampoules containing the $\mathrm{Sn}$-Se alloy were purged with argon and sealed under a vacuum of $10^{-5}$ Torr. In order to achieve well-homogenized samples before carrying out the solidification experiments, the alloys were processed in a rocking furnace at $860{ }^{\circ} \mathrm{C}$ for four hours.

The DSC technique allows one to determine differences in heat flow into a sample and a reference sample, while both samples are subjected to a programmed temperature evolution ${ }^{14}$. During the eutectic solidification, this heat flow difference increases due to the latent heat release. To carry out such a thermal analysis, a special procedure was utilized. Due to the easy selenium volatility that could damage the DSC unit as well as lead to inaccurate measurements, small amounts of the eutectic alloy, from 20.0 to $116.0 \mathrm{mg}$, were again sealed in small quartz ampoules 6.0 $\mathrm{mm}$ OD x $5.0 \mathrm{~mm}$ ID and $8.0 \mathrm{~mm}$ long, under vacuum and purged again with argon. After preparation, these samples were inserted directly into the sample carrier of the Netzsch STA 404 thermal analysis equipment. Before doing the DSC experiments, the thermal analysis unit was calibrated using standard samples. The eutectic transformation tem- 
perature was assumed to be the temperature at the start of the DSC peak.

The alloy was subjected to steady state directional growth in the vertical Bridgman-Stockbager crystal growth equipment with a provision for measurements of thermal profile in the sample during the growth process. This directional solidification unit consisted of hot and cold zones made of Kanthal heating devices, isolated by an adiabatic zone. The samples were lowered from the hot unit to the cold unit to allow the directional solidification at growth rates varying from $2.8 \times 10^{-4}$ to $14.0 \times 10^{-4} \mathrm{~cm} / \mathrm{s}$. Ingots 6.0 $\mathrm{cm}$ long and $0.8 \mathrm{~cm}$ in diameter were placed inside a quartz ampoule. To determine the temperature evolution during the solidification, three small depressions $1.0 \mathrm{~mm}$ in diameter were made transversely through the center of the quartz ampoule to locate the thermocouples. Figure 1 presents the samples diagram, including the position of the thermocouples.

The temperature evolution obtained by the thermocouples, the distance between each thermocouple and the slope of the cooling curves, allow one to calculate the growth rate, the thermal gradient at solid/liquid interface and the interface undercooling. The temperature evaluation was obtained by using three fine Chromel-Alumel thermocouples (100 $\mu \mathrm{m}$ in diameter) with exposed junctions, which were connected to a high precision computerized data acquisition system. The thermocouples were placed $2.0 \mathrm{~cm}$ away from each other. In order to improve the thermal contact in the thermocouple positions, the gap between the thermocouple junctions and the quartz wall was filled with a mixture of graphite powder and alcohol. The alcohol

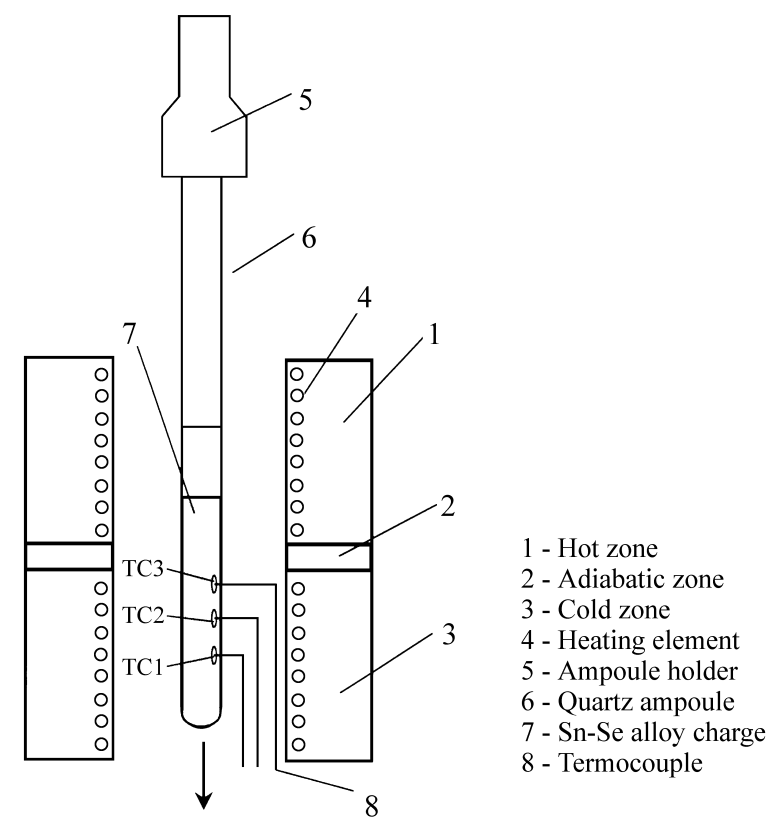

Figure 1. A schematic drawing of the directional solidification apparatus including the provision for the sample temperature measurements. volatilization with an increase in the temperature led to a more efficient heat transfer process between the quartz and the thermocouples.

The effect of the growth conditions on the eutectic microstructure was found by taking cross section samples at several locations along the ingot. The sections were mounted with wax on a brass matrix. The samples were mechanically polished and then, chemically polished in a solution of $100 \mathrm{~mL}$ of absolute ethanol and $2 \mathrm{~g}$ of iodine. The microstructure analysis and the spacings measurements were found by using an optical microscope. As the eutectic microstructure consists of very small $\mathrm{SnSe}$ and $\mathrm{SnSe}_{2}$ lamellas, an SEM study was performed. Longitudinal cut sections were also observed by using optical and scanning electron microscopes.

\section{Results and Discussion}

The reason for performing the thermal analysis of the Sn-Se eutectic alloy was connected to the evaluation of the equilibrium temperature of the transformation liquid $\rightarrow$ $\mathrm{SnSe}+\mathrm{SnSe}_{2}$ as a significant discrepancy is verified among the results obtained by several authors ${ }^{16}$. In order to eliminate the evident inconsistencies in these earlier investigations, the eutectic equilibrium temperature was determined during the heating cycle of the thermal analysis, as in the cooling cycle the eutectic temperature is a function of the growth rate ${ }^{17-20}$. Since the sample was very small, it was virtually impossible to measure its growth rate. However, the growth rate is a function of the cooling rate and the thermal gradient. Figure 2 presents typical heating and cooling cycles obtained during the experiments.

As the thermal contact inside the small quartz ampoule is modified by the melting and solidification of the sample, several measurements were performed for each condition of heating or cooling rate. The results of the heating cycles led to a eutectic equilibrium temperature equal to $627.8 \pm$ $0.1{ }^{\circ} \mathrm{C}$, which is closed to the value found by Feutilais et

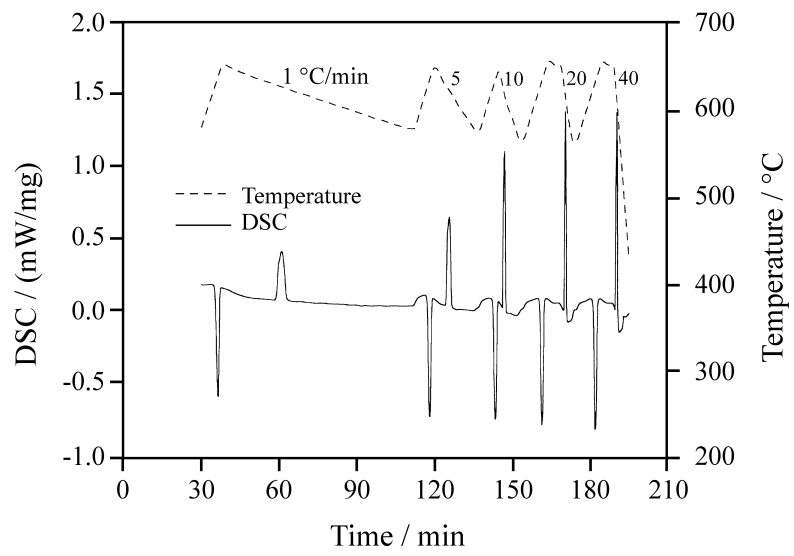

Figure 2. Typical heating and cooling cycles of an Sn-Se eutectic alloy obtained during a DSC analysis. 
$a l .{ }^{16}$. However, if such a value is compared to other results presented by Feutilais et al. ${ }^{16}$, the difference can reach as much as $12{ }^{\circ} \mathrm{C}$. During the cooling cycle, it was possible to observe that the growth rate was directly connected to the cooling conditions, as an increase in the cooling rate caused a decrease in the eutectic temperature, as shown in Fig. 3. In the analysis of a $45.0 \mathrm{mg}$ sample, when the cooling cycle was set at $1.0{ }^{\circ} \mathrm{C} / \mathrm{min}$, the temperature of the eutectic reaction was found to be $627.0 \pm 0.1^{\circ} \mathrm{C}$. For a cooling rate equal to $40{ }^{\circ} \mathrm{C} / \mathrm{min}$, the eutectic transformation occurred at a temperature of $625.1 \pm 0.1{ }^{\circ} \mathrm{C}$. Certainly, the undercooling is also a function of the sample characteristics, as the growth rate during the solidification depends on the heat transfer conditions. The cooling process is controlled by a number of parameters, including the sample mass and the thermal contact between the sample and the quartz ampoule. When the sample mass was increased to $116.0 \mathrm{mg}$, the eutectic temperature changed. At a cooling rate of $40.0^{\circ} \mathrm{C} / \mathrm{min}$, the eutectic reaction temperature changed to $626.2 \pm 0.1{ }^{\circ} \mathrm{C}$. An increase in the sample mass leads to a higher solidification time, and hence, the growth rate can significantly change. While the outer layer of the sample solidifies rapidly, the internal part of the same sample solidifies at a lower rate.

The directional solidification experiments provide interesting results. By using the distance between the thermocouples and the cooling curves, one is easily able to determine the growth rate as well as the temperature gradients at the solid/liquid interface. Also, as the solidification could be assumed to be in a steady state, the cooling curve slopes were used to confirm the results associated with the thermal gradients ${ }^{21}$. In spite of the fact that the growth rate can usually diverge from the ampoule lowering rate during a directional solidification in a Bridgman-Stockbarger furnace, in our case, the differences between both values were negligible. However, we found that an increase in the

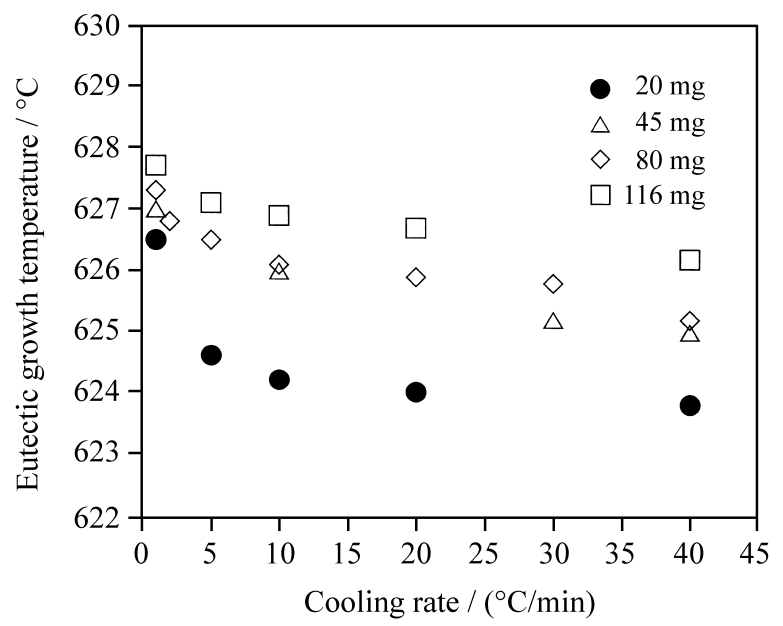

Figure 3. Evolution of the eutectic growth temperature with the cooling rate obtained in a DSC Analysis for several mass samples. lowering rate led to an increase in such a difference. The results obtained from cooling curves and microstructure examination gave rise to the plot presented in Fig. 4 which provides the value of $\mathrm{K}_{2} / \mathrm{K}_{1}=1.54 \times 10^{-11} \mathrm{~cm}^{3} / \mathrm{s}$ (Eq. 2). These results are in excellent agreement with previous measurements obtained during directional growth of the Sn-Se eutectic alloy ${ }^{12}$.

The thermal gradient calculated from the sample temperature evolution varied from $20.0^{\circ} \mathrm{C} / \mathrm{cm}$ to $40.0^{\circ} \mathrm{C} / \mathrm{cm}$. A comparison among several directionally solidified samples, processed at different thermal gradients, resulted in the fact that the $\mathrm{SnSe}-\mathrm{SnSe}_{2}$ eutectic microstructure slightly increases its regularity as the thermal gradient at the solid/liquid interface increases. However, no change was observed in the distribution of individual lamellar spacings. It is likely that, changes in the thermal gradient were not significant enough to alter the lamellar spacing selection.

In addition to the growth rate and the thermal gradient, the use of thermocouples to follow the thermal behavior of an ingot under directional solidification allows one to evaluate the eutectic growth temperature. According to classical eutectic growth theory, this temperature should decrease with an increase in the growth rate. The growth temperature and the location of the solid/liquid interface were not easily found from the cooling curves as the slope was not always abruptly changed. Consequently, to measure such a temperature, a large number of experiments for each growth condition was required. The growth temperature was also studied by comparing the microstructure obtained in the directional solidification experiments to microstructure obtained in DSC analysis. The microstructure comparison comprised the study of the interphase spacings only. The spacings obtained during directional growth were compared to the ones found in the microstructure of DSC samples obtained at several levels of cooling

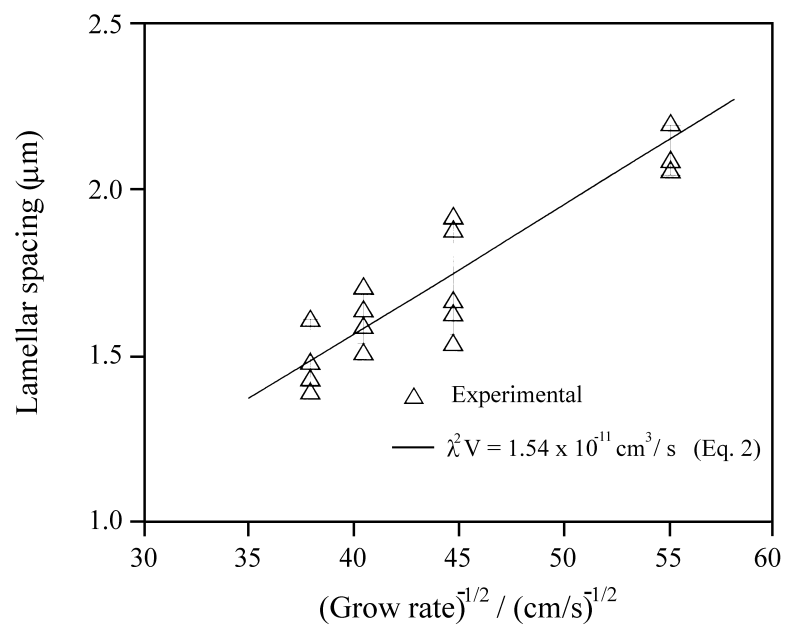

Figure 4. Lamellar spacing as a function of the inverse square root of the growth rate obtained by computing the cooling curves data. 
rate. Although the growth conditions were different, as solidification in the DSC was not directional, it was found that the average spacings were controlled by the cooling rate. The results of DSC and directional solidification analyses led to the evolution of the eutectic growth temperature as a function of the growth rate. Figure 5 presents a plot of the eutectic growth temperature versus the square root of the growth rate. As the growth rate increases, the growth undercooling also increases, which allows one to find the value of the constant in Eq. 3. Also, extrapolation to zero growth rate produces equilibrium eutectic temperature of $628.4 \pm 0.3^{\circ} \mathrm{C}$, which is in good agreement with the eutectic temperature found in the heating cycles of the DSC analysis and with the results obtained by Feutilais et al. ${ }^{16}$.

By observing the plot of the eutectic growth undercooling as a function of the square root of the growth rate, the value of the constant $\mathrm{K}_{3}$ in Eq. 3 was found to be equal to $65.0 \mathrm{Ks}^{1 / 2} \mathrm{~cm}^{-1 / 2}$. By analyzing the value of the constants $\mathrm{K}_{1}, \mathrm{~K}_{2}$ and $\mathrm{K}_{3}$ for several systems (Eqs. 1 to 4 ) presented by Kurz and Fisher ${ }^{4}$, one is able to verify that the values obtained in the present study are quite reasonable.

The growth behavior of the eutectic $\mathrm{SnSe}-\mathrm{SnSe}_{2}$ is very intriguing. In spite of the fact that the lamellar spacing is not unique and a wide distribution of values is found for a given growth rate, the $\mathrm{Sn}$-Se eutectic microstructure is very regular. Figure 6 presents typical microstructures of a directionally solidified $\mathrm{Sn}-\mathrm{Se}$ eutectic sample. Nevertheless, the entropies of fusion of both $\mathrm{SnSe}$ and $\mathrm{SnSe}_{2}$ compounds are very large ${ }^{16}$. This fact would lead to the assumption that the $\mathrm{Sn}$-Se eutectic alloy grows without an easy-branching behavior $^{22}$. However, an aligned and regular eutectic microstructure obtained from the directional solidification of the Sn-Se system shows the opposite. Explanation of such a phenomenon can be found in the Podolinsky et al. investigation $^{23}$. In solutions, a faceted component can become non-faceted and vice-versa, as a function of the effect of the other component ${ }^{23}$.

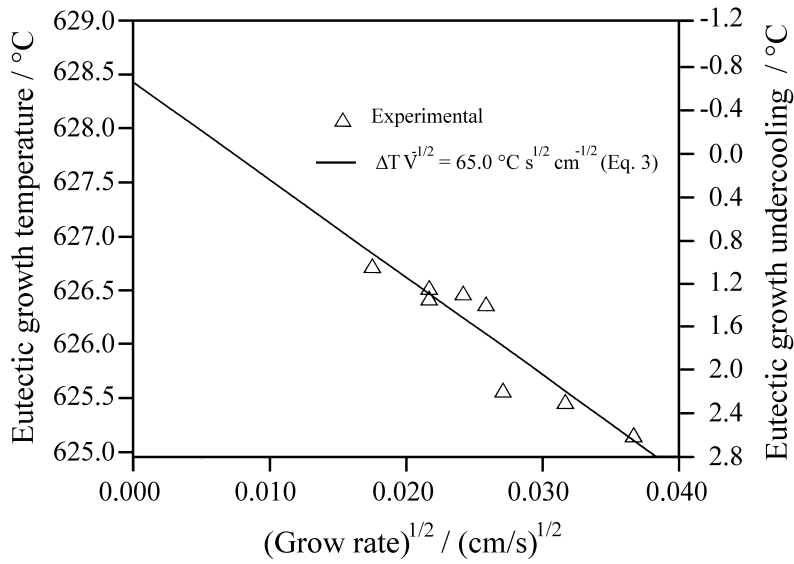

Figure 5. Variation of the eutectic growth temperature with the square root of the growth rate.
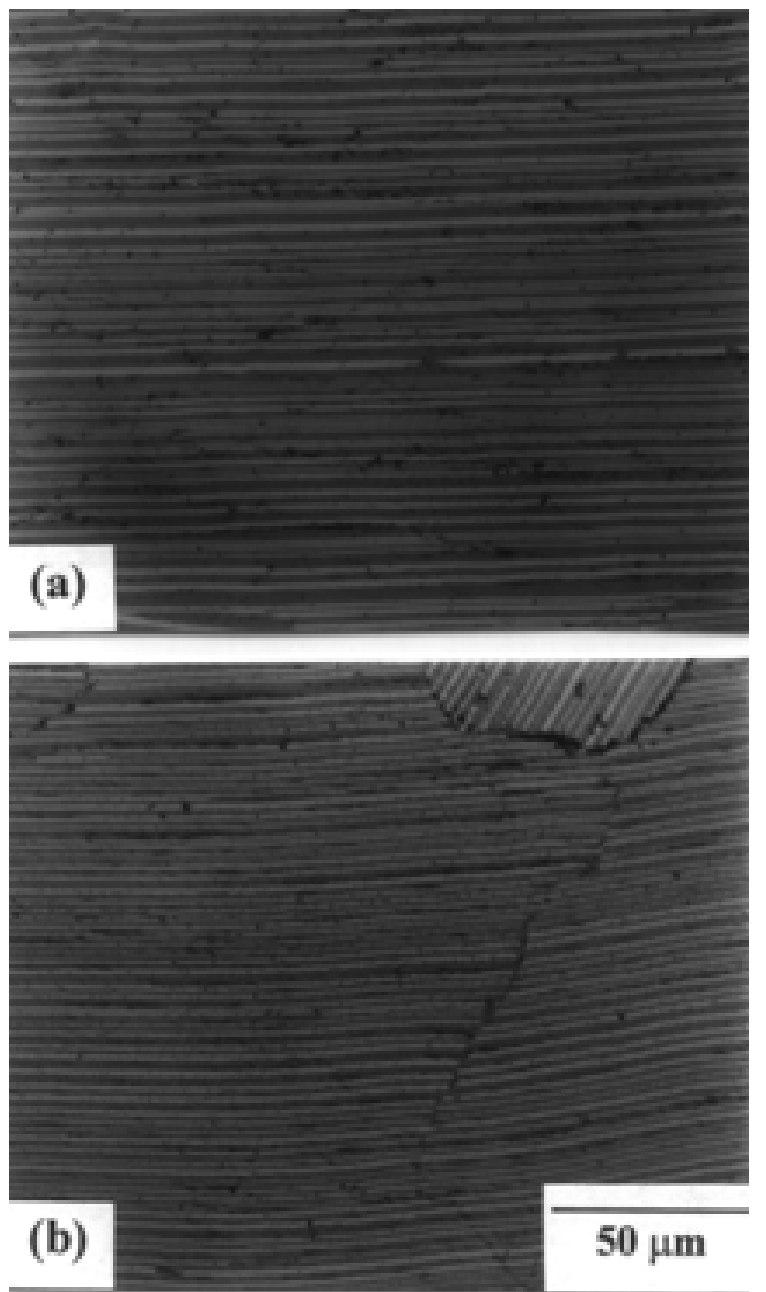

Figure 6. Typical microstructures obtained from directionally solidified Sn-Se eutectic samples: (a) longitudinal view; (b) cross view.

\section{Conclusions}

Thermal analysis and directional growth experiments were carried out on the Sn-Se eutectic alloy, and the effect of the growth rate on the eutectic growth temperature was investigated. Based upon the results obtained, it can be affirmed that the effect of the growth rate on the eutectic microstructure is in good agreement with the theory, i.e., the lamellar spacing is proportional to the inverse of the square root of the growth rate and a relationship between $\lambda$ and $\mathrm{V}$ was achieved. The equilibrium eutectic temperature was found to be $627.8 \pm 0.1^{\circ} \mathrm{C}$, which is in good agreement with recent results found in the literature. As predicted by the Jackson and Hunt theory ${ }^{1}$, it was verified that the eutectic growth temperature depends on the growth rate. By using different values of thermal gradients, it was observed that an increase in the temperature gradient at the solid/liquid interface produces a slight improvement in the microstructure regularity. Although the entropy of fusion of the $\mathrm{SnSe}$ and $\mathrm{SnSe}_{2}$ compounds found in the literature 
are very high, and hence an irregular eutectic growth would be expected, in all the growth conditions employed, the microstructure remained regular.

\section{Acknowledgments}

The authors would like to thank CNPq, FAPESP and FAEP-UNICAMP for their financial support.

\section{References}

1. Jackson, K.A.; Hunt, J.D. Trans. AIME, v. 236, p. 1129, 1966.

2. Tiller, W.A. Liquid Metals and Solidification, ASM, Metals Park, OH, p. 276, 1958.

3. Elliot, R. International Metals Reviews, v. 22, p. 161, 1977.

4. Kurz, W.; Fisher, D.J. International Metals Reviews, v. 24, p. 177, 1979.

5.Zener, C. Trans. AIME, v. 167, p. 550, 1946.

6. Trifonova, E.; Yanchev, I.Y.; Manou, P.; Kambas, K.; Anagnostopoulos, A.N. Journal of Materials Science, v. 31, p. 3647, 1996.

7. Bhatt, V.B.; Gireesan, K.; Desai, C.F. Journal of Materials Science Letters, v. 11, p. 380, 1992.

8. John, K.J.; Pradeep, B.; Mathai, E. Journal of Materials Science, v. 29, p. 1581, 1994.

9. Bhatt, V.B.; Gireesan, K.; Pandya, G.R. Journal of Crystal Growth, v. 96, p. 649, 1989.
10. Siddiqui, S.S.; Desai, C.F. Thin Solid Films, v. 239, p. 166, 1994.

11. Bhatt, V.B.; Gireesan, K.; Desai, C.F. Indian Journal of Pure \& Applied Physics, v. 29, p. 27, 1991.

12. Aguiar, M.R.; Caram, R. Journal of Crystal Growth, v. 166, p. 398, 1996.

13. Aguiar, M.R.; Caram, R. Journal of Crystal Growth, v. 174, p. 70, 1997.

14. Kaisersberger, E. Labor Praxis, v. 9, p. 704, 1990.

15. Overfelt, R.A.; Matlock, C.A.; Wilcox, R.C. Journal of Crystal Growth, v. 147, p. 403, 1995.

16. Feutilais, Y.; Majid, M.; Legendre, B.; Fries, S.G. Journal of Phase Equilibria, v. 17, p. 40, 1996.

17. Dong, L.; Jones, H. Scripta Metallurgica et Materialia, v. 25, p. 2855, 1991.

18. Dong, L.; Jones, H. Zeitschrift für Metallkunde, v. 83, p. 244, 1988.

19. Hughes, I.R.; Jones, H. Journal of Materials Science, v. 11, p. 1781, 1976.

20. Magnin, P.; Kurz, W. Zeitschrift für Metallkunde, v. 79, p. 282, 1988.

21. Burden, M.H.; Hunt, J.D. Journal of Crystal Growth, v. 22, p. 99, 1974.

22. Jackson, K.A. Liquid Metals and Solidification, ASM, Metals Park, OH, p. 174, 1958.

23. Podolinsky, V.V.; Taran, Y.N.; Drykin, V.G. Journal of Crystal Growth, v. 96, p. 445, 1989. 